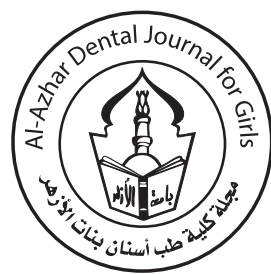

\title{
Stem Cells and Their Potential Effect on Irradiated Submandibular Salivary Glands in Mice
}

\author{
Seham I. Hallool ${ }^{*}$, Mona H. Farid ${ }^{2}$, Samah S. Mehanny ${ }^{3}$, Hanan F. Abdel Hamid ${ }^{4}$
}

Codex : 05/2001
azhardentj@azhar.edu.eg
http://adjg.journals.ekb.eg
DOI: $10.21608 /$ adjg.2019.7619.1099

\section{KEYWORDS}

BM-MSCs,

submandibular salivary glands, radiation, immunofluorescence, anti- $\alpha$ - amylase 1 .

\begin{abstract}
Purpose: the objective of the present study was to evaluate the effect of BM-MSCs on irradiated submandibular salivary glands in mice and to determine at which stage post-irradiation the cells should be transplanted. Materials and methods: 58 adult male mice were used, 10 for preparation of PKH-26-labeled BM-MSCs and the remaining 48 mice were divided into: control group (GI) consisted of 6 mice without radiation and experimental groups consisted of 42 mice irradiated with a single dose of 15Gy. They were randomly divided into three groups: GII (+ve control): 18 irradiated not treated mice and two treated groups each consisted of 12 irradiated mice and treated with intraglandular injection of labeled BM-MSCs, 24 hours (GIII) and at day 11 (GIV) post-radiation. Animals were sacrificed at day 6, day 30 and day 90 post-radiation. The submandibular glands were collected, one gland was routinely prepared for histological examination using H\&E staining and the other was prepared for immunohistochemical study using immunofluorescence staining with anti- $\alpha$-amylase 1 antibody. Results: BM-MSCs produced marked improvement in salivary gland tissue architecture, histology of parenchymatous and stromal structures and glands function indicated by marked increase in $\alpha$ amylase 1 production compared with +ve control group, with GIII showed greater improvement than GIV. Conclusions: It could be concluded that: intraglandular transplantation of BM-MSCs could successfully regenerate radiation-induced submandibular salivary gland damage and restore the gland function. The early transplantation of BM-MSCs 24 hours post-radiation was markedly more beneficial than the transplantation at day 11 post-radiation.
\end{abstract}

- Paper extracted from Doctor thesis titled (Stem Cells and Their Potential Effect on Irradiated Submandibular Salivary Glands in Mice).

1. Assistant Lecturer of Oral and Dental Biology, Faculty of Dental Medicine for Girls, Al-Azhar University.

2. Professor of Oral and Dental Biology, Former Dean of the Faculty of Dental Medicine for Girls, Al-Azhar University.

3. Professor of Oral and Dental Biology, Faculty of Dentistry, Cairo University.

4. Assistant Professor of Biochemistry, National Center for Radiation Research and Technology (NCRRT), Atomic Energy Authority, Cairo.

*Corresponding author Email: sehamhallool@gmail.com 


\section{INTRODUCTION}

Salivary glands are responsible for secretion of approximately one and half liter of saliva daily. Saliva plays a pivotal role in moistening the oropharyngeal mucosa which is essential for speech, taste sensation, mastication, preparing the bolus of food and deglutition ${ }^{(1)}$.

In addition, saliva plays an essential role in the maintenance of teeth integrity and has several constituents with digestive, protective and antimicrobial effects ${ }^{(2)}$. Saliva also contains growth factors e.g. epidermal growth factor which enhances ulcers healing ${ }^{(2,3)}$. For these reasons, any dysfunction or cessation to saliva production greatly affects oral and general health and comfort ${ }^{(1,4)}$.

Moreover, several systemic conditions can produce hyposalivation and xerostomia including; aging, uncontrolled diabetes mellitus, hypertension, medications ${ }^{(5)}$. However, one of the most severe conditions that cause severe xerostomia is radiotherapy for head and neck cancer ${ }^{(6)}$.

According to statistics that were done in 2016; squamous cell carcinoma in head and neck region is the fifth most common malignancy all over the world ${ }^{(7)}$. Most head and neck cancer are routinely treated with radiotherapy, either alone or together with chemotherapy, surgery, or both ${ }^{(8)}$. Before the introduction of updated radiotherapy modalities, more than $80 \%$ of survivors of patients receiving radiotherapy suffered from dry mouth syndrome which is irreversible and a life-long problem (9- 11). Although the use of the advanced modalities, radiotherapy still causes xerostomia in high percentage $^{(12-14)}$, approximately $40 \%{ }^{(12)}$.

Although salivary acinar cells are highly differentiated slowly proliferating cells, they are highly radiosensitive ${ }^{(15,16)}$. The serous acini are affected by radiation more quickly than the mucous acini and the ducts ${ }^{(15-17)}$. Two main phases of salivary gland response to radiation are described: early and late. The early phase is noticed up to day 10 post-radiation ${ }^{(18)}$ with the observed morphological changes are maximal at day 6 post- radiation ${ }^{(18-20)}$. Then, signs of tissue regeneration start to appear at day 10 post- radiation. At day 40 post- radiation, the gland architecture is greatly improved. The second, late phase is observed at day 90 post- radiation with severe glandular destruction, marked decrease in acinar cells' number, edema and disseminated mononuclear infiltration ${ }^{(18)}$.

On the other hand, several axes have been performed for management of radiation-induced xerostomia including: preventive measures and treatment of radiation induced xerostomia. The preventive measures aiming for salivary gland protection using intensity modulated radiotherapy (IMRT), adaptive modulated therapy (AMT), salivary gland transfer and radioprotectors ${ }^{(6)}$.

Moreover, treatment of radiation induced xerostomia includes palliative treatments, salivary gland stimulation using sialogogues or acupuncture, and regeneration of the damaged salivary glands. Salivary gland regeneration can be achieved using alternative treatment approaches including growth factors, gene therapy, tissue engineering, or stem cell-based therapy ${ }^{(6,21,22)}$.

Mesenchymal stem cells (MSCs) are multipotent stem cells. The first known source for them was bone marrow (BM-MSCs). Today, there are several sources for their isolation including the adipose tissue, umbilical cord blood, umbilical cord membrane, placenta, dental pulp and periodontal ligament ${ }^{(23-25)}$.

Furthermore, BM-MSCs like other MSCs can act by two mechanisms: their differentiation potential and secretion of soluble factors. MSCs have the potential to differentiate into cells of the three germ layers as reported by several in vitro and in vivo studies (23-25). In addition, they secrete many soluble factors which have several paracrine effects on the nearby cells which enhance tissue repair. Their secreted factors can also strongly regulate the immune responses by affecting all the cells of the immune system to produce an anti-inflammatory phenotype ${ }^{(23)}$. 
Moreover, MSCs are now the most common type of stem cells applied clinically. Till now, MSCs from different sources have been used clinically to treat several degenerative and immune-related diseases with high effectiveness and safety ${ }^{(23-25)}$.

Since radiation-induced damage to salivary stem/progenitor cells is considered as the cause for the resultant xerostomia, stem cells transplantation can greatly enhance salivary gland regeneration ${ }^{(26)}$. It has been demonstrated that direct intraglandular transplantation of BM-MSCs, 24 hours post-radiation, can regenerate the damaged gland and restore its function by differentiation into saliva producing cells as well as secreting paracrine factors ${ }^{(27)}$.

\section{MATERIAL AND METHODS}

\section{Animals:}

Fifty eight adult male mice (weighing 18-25 g, 8-12 weeks old) were used in this study. Animal handling followed the rules and regulations of the animal experimental studies that were approved by ethical committee including their facilities, diet and method of scarification.

Ten mice were used for BM-MSCs preparation and the remaining forty eight mice were randomly divided into: A control group (group I), consisted of 6 non irradiated mice and experimental groups, consisted of 42 irradiated mice. They were randomly divided, after radiation, into three groups, including; group II (+ve control): consisted of 18 irradiated non treated mice, group III: consisted of 12 irradiated mice, treated with intraglandular injection of labeled BM-MSCs into their submandibular salivary glands, 24 hours post-radiation and group IV: consisted of 12 irradiated mice, treated with intraglandular injection of labeled BM-MSCs into their submandibular salivary glands, at day 11 postradiation.

\section{Isolation and expansion of mice BM-MSCs:}

The tibias and femurs of the mice were collected, bone marrow samples were gathered from them and BM-MSCs were isolated and then identified us- ing flow cytometric and phenotyping analysis ${ }^{(28,29)}$. BM-MSCs were then labeled using PKH-26 Red Fluorescent Cell Linker Kit (Sigma Aldrich, St. Louis, MO, USA).

\section{Irradiation process:}

The adult male mice were generally anaesthetized with thiopental sodium (Egyptian Pharmaceutical International Company (EPICO), was purchased from local market), ultra short acting barbiturate, $(60 \mathrm{mg} / \mathrm{Kg})$ injected intra-peritoneally (i.p.) ${ }^{(30,31)}$. Then, the mice head and neck region received 15 Gray (15Gy) gamma radiation, using lead shield for the rest of the body ${ }^{(11,27,32)}$.

\section{Intraglandular transplantation of BM-MSCs:}

24 hours post-radiation ${ }^{(27)}$, the mice of both group II and group III were generally anaesthetized with i.p. injection of thiopental sodium, $(60 \mathrm{mg} / \mathrm{Kg})$ ${ }^{(30,31)}$. A vertical incision of the neck was made and the skin was retracted gently to expose submandibular salivary glands. Then, each submandibular gland of the mice of group III was directly injected with $1 \times 10^{5} \mathrm{PKH}-26$ labeled BM-MSCs in $50 \mu 1$ of PBS, while the submandibular glands of the mice of group II were injected with $50 \mu 1$ of PBS alone ${ }^{(27,33)}$.

After injection, the surgical wound was sutured using coated VICRYL ${ }^{\circledR}$ braided suture (50) (A synthetic, absorbable, surgical suture, was purchased from local market), then covered with Penicillin G powder antibiotic. At day 11 post-radiation ${ }^{(34)}$, the mice of group IV were anaesthetized and injected with BM-MSCs by the same way that was mentioned before.

\section{Specimens collection:}

At day 6 post-radiation (D6), 6 mice from group II were anaesthetized then sacrificed separately by cervical dislocation. A vertical incision of the neck was made and the skin was retracted gently to expose submandibular salivary glands, then the glands were dissected. At the same way, the glands of 6 mice from each group were collected at both 
day 30 (D30) and day 90 (D90) post-radiation. For each sacrificed mouse, one gland was prepared and histologically examined, while the other gland was prepared for immunohistochemical study using immunofluorescence staining.

\section{Specimens preparation:}

\section{a. Preparation of the specimens for histological examination:}

The specimens were routinely fixed, dehydrated, cleared and paraffin wax embedded. Then 4 micron thickness sections were cut, stained by $\mathrm{H} \& \mathrm{E}$ stain and examined by light microscope at different magnifications.

\section{b. Preparation of the specimens for immunohisto- chemical study using immuonofluorescence stain- ing to detect $\alpha$-amylase 1 secretion:}

Paraffin tissue sections were deparaffinized and rehydrated then antigen retrieval was performed. The sections then were incubated with blocking buffer for $1 \mathrm{~h}$, then incubated with the primary antibody, rabbit polyclonal anti- alpha amylase 1 (AMY1A) (were purchased from (Chongqing Biospes Co., Ltd, China). Detection of bound antibodies was done by Alexa Fluor secondary antibodies (were purchased from (Abcam Cambridge, MA, USA). Nuclei were stained with DAPI (4,6-diamino-2-phenyllindole), was purchased from (Abcam Cambridge, MA, USA). Slides were mounted and examined using Nikon fluorescence microscope under blue, green and red channels. Fluorometric analysis then was performed ${ }^{(35,36)}$.

\section{Statistical analysis:}

Computerized image analysis was used to assess the percentage of $\alpha$-amylase 1 secretion positive surface areas within the immunofluorescent stained sections of all groups. The positive surface areas appeared as green areas. The results were then collected, tabulated and statistically analyzed.
Statistical analysis was performed using a commercially available software program (SPSS 19; SPSS, Chicago, IL, USA). As data was parametric, ANOVA test was used for evaluation of values, followed by Tukey's post hoc test.

\section{RESULTS}

\section{A. Histological results:}

Examination of H\&E stained sections from submandibular salivary glands of group II at different time intervals (D6, D30 and D90) post-radiation revealed that the most severely destructed glands appeared at D90 with the most severe histopathologic changes, acinar atrophy and shrinkage and extremely wide interlobular and inter acinar spaces; compared with the other intervals, especially D30 which showed the best gland architecture with the least histopathologic changes. Fig. (1-A)

Comparing H\&E stained sections from submandibular salivary glands of group II, group III and group IV at D90 post-radiation revealed that group III showed relatively normal gland architecture with compact acinar arrangement, normal inter acinar and interlobular spaces, markedly the most preserved acinar and ductal architecture and cell lining and the most clearly apparent mitotic figures while the glands of group II appeared markedly the most severely destructed. Fig. (1-B)

\section{B. Immunohistochemical results for detection of $\alpha$-amylase 1 secretion (immuonofluorescence staining):}

Fluorescence microscopic examination of immunofluorescent stained sections from the submandibular salivary glands of group II at different intervals (D6, D30, D90) post-radiation revealed +ve reaction of the tissues to $\alpha$-amylase 1 secretion. The reaction was the highest at the 6th day post-radiation, but less than the reaction that was expressed in the control group. The least reaction was found at day 90 post-radiation. Fig. (2-A) 
Comparing the immunofluorescent stained sections from submandibular salivary glands of group II, group III and group IV at D90 post-radiation revealed that group II (GII D90) showed the least reaction to $\alpha$-amylase 1 secretion, while it was the highest in group III. Fig. (2-B)

\section{Statistical analysis:}

The highest mean value of $\alpha$-amylase 1 surface area was recorded in control group (GI), whereas the lowest mean value was recorded in group II (ir-

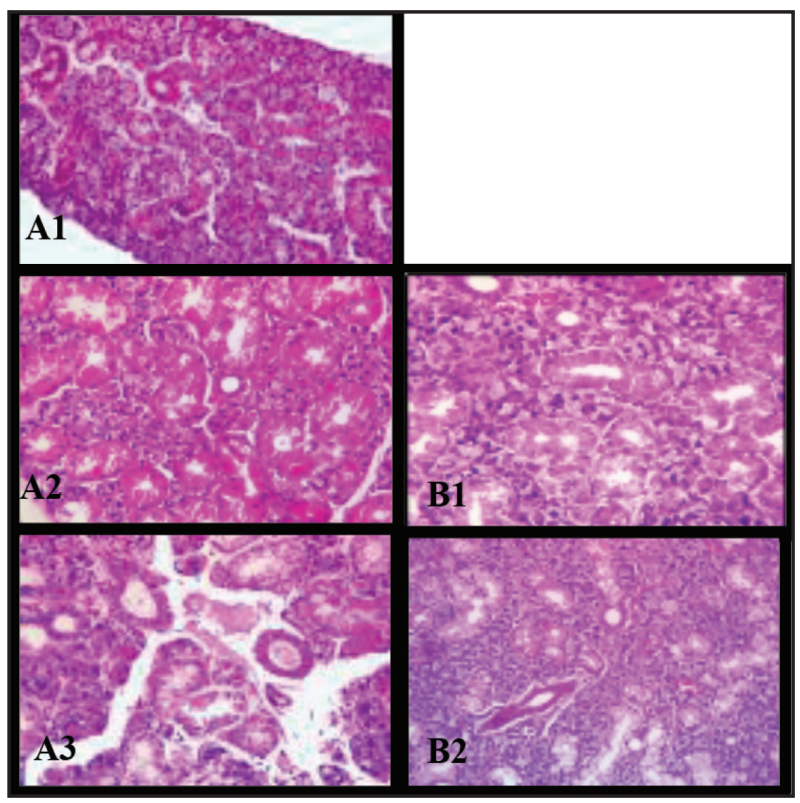

Figure (1) A photomicrograph of $H \& E$ stained sections (x400) from the submandibular salivary glands of experimental groups. (A): comparing sections of group II at D6 (A1), D30 (A2) and D90 (A3) revealing that D90 showed the most severely destructed glands with the most severe histopathologic changes, acinar atrophy and shrinkage and extremely wide interlobular and inter acinar spaces. (A3\& B): comparing sections of group II (A3), group III (B1) and group IV (B2) at D90 revealing that GIII showed relatively normal gland architecture with compact markedly the most preserved acinar and ductal architecture and cell lining and the most clearly apparent mitotic figures. radiated group) day 90 post-radiation (GII D90). ANOVA test revealed that the difference between all groups was extremely statistically significant. Tukey's post hoc test revealed that GI was significantly higher than all other groups, while GII D90 was significantly lower than all other groups. However, there was no significant difference between groups: GII D6, GIII D30, GIV D30 and GIII D90. Moreover, there was no significant difference between groups: GII D30, GIV D30 and GIV D90. (Fig. 3)

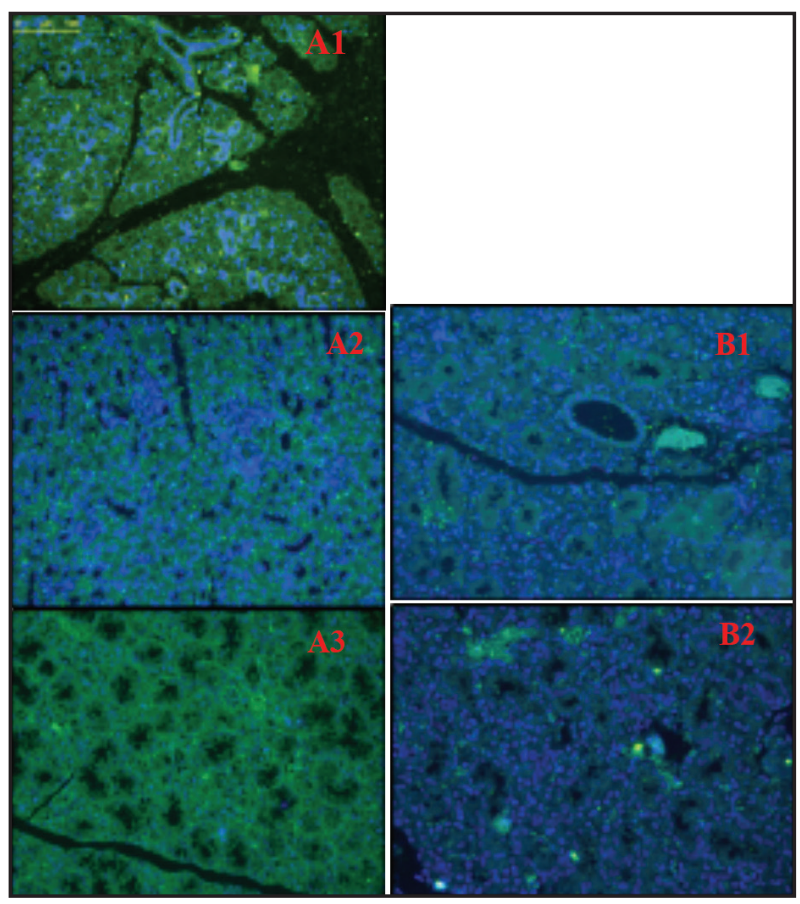

Figure (2) A photomicrograph of IF stained sections (x200) from the submandibular salivary glands of experimental groups. (A): comparing sections of group II at D6 (A1), D30 (A2) and D90 (A3) showing +ve reaction to $\alpha$-amylase 1 secretion (green areas) at D6, less reaction at D30 and the least reaction at D90. (A3\&B): comparing sections of group II (C1), group III (C2) and group IV (C3) at D90 showing the least reaction to $\alpha$-amylase 1 secretion in group II, while the highest in group III. DAPI (blue). 


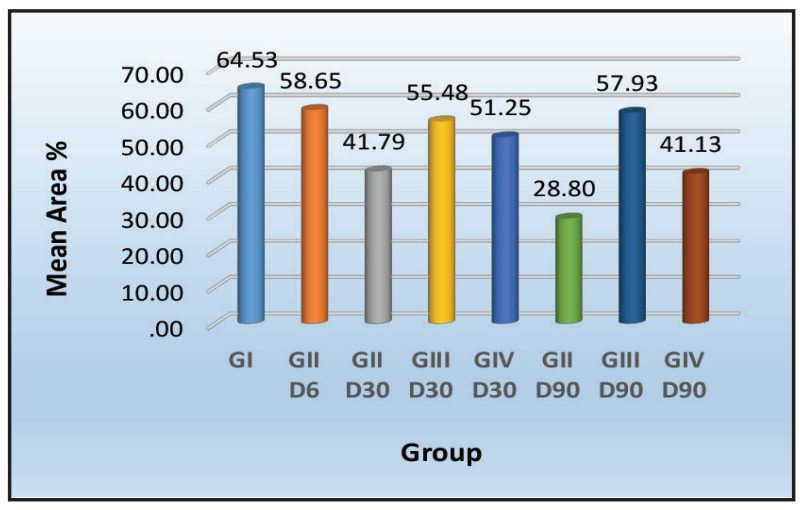

Figure (3) Column chart showing mean $\alpha$-amylase 1 positive surface area value $(\%)$ in all groups

\section{DISCUSSION}

The aim of this study was based on the recommendation of a previous study to perform further investigations to determine at which stage postradiation the BM-MSCs should be transplanted ${ }^{(27)}$. In this study, single intraglandular injection of BMMSCs was done either $24 \mathrm{~h}$ post-radiation ${ }^{(27)}$ or at day 11 post-radiation ${ }^{(34)}$. These chosen dates were selected to be either nearly at the beginning or at the end of the early phase of post- radiation gland reaction ${ }^{(18,34)}$ to assess whether the time of BM-MSCs transplantation could affect the gland regeneration.

In the foregoing study, day 6 was selected for specimens collection to confirm the early phase of radiation induced salivary glands damage which has been estimated to be maximal at day 6 postradiation ${ }^{(18-20)}$.

Moreover, day 30 was selected for specimen collection in accordance with previous studies ${ }^{(27,34)}$, to study the intermediate phase of radiation induced damage of the salivary secretory tissue in which some tissue regeneration and attempts towards recovery were observed ${ }^{(6,18)}$.

In addition, day 90 was selected in agreement with previous studies ${ }^{(8,27)}$, to study the late phase of radiation induced damage of the salivary secretory tissue and to assess the beneficial effect of the injected BM-MSCs on the irreversibly damaged salivary gland tissue.
The histological results of the present study revealed that the submandibular glands of the irradiated non treated group (group II) appeared most severely destructed at D90 compared with the other intervals, especially D30 which showed the best gland architecture with the least histopathologic changes. These findings were closely agreed with several previous studies ${ }^{(18,27)}$.

Moreover, comparing $\mathrm{H} \& \mathrm{E}$ stained sections from the glands of group II, group III and group IV revealed that group III showed the best gland architecture which appeared relatively normal with markedly the most preserved acinar and ductal architecture and cell lining and mild histopathologic changes compared with the other two groups, especially group II. The glands of group II appeared the most severely destructed. These foregoing observations revealed that intraglandular transplantation of BM-MSCs 24 h post- radiation greatly enhanced regeneration of irradiated salivary glands. These findings agreed with a previous study which concluded that intraglandular transplantation of BM-MSCs 24 $\mathrm{h}$ post-radiation provided regeneration of glandular tissues and gland function to $80-90 \%$ of non- irradiated controls, at day 90 post- radiation. They reported that BM-MSCs exerted this effect through their paracrine actions and their potential to transdifferentiate into salivary epithelial cells ${ }^{(27)}$.

An interesting observation related to the time of BM-MSCs transplantation revealed that less improvement in the submandibular glands of mice in group IV than those in group III. These finding suggested that the early transplantation of BM-MSCs $24 \mathrm{~h}$ post-radiation, i.e. almost with the beginning of early phase of radiation damage, was more beneficial for tissue regeneration than the transplantation at day 11 post-radiation, i.e. after the end of the early phase. This could be explained as, the transplanted BM-MSCs 24 hours post-radiation with their powerful anti- inflammatory and immunomodulatory functions ${ }^{(23,25)}$ could able to decrease the inflammatory reaction that occur immediately post-radiation. This in turn could reduce the immune-mediated 
destruction of the parenchyma ${ }^{(20)}$, compared with the transplanted cells after the end of the early phase in which the inflammatory reaction has already established and produced destruction of the parenchyma.

On the other hand, immunohistochemical studying of specimens using immunofluorescence staining was performed in this study and the results were analyzed statistically to assess and compare the percentage of $\alpha$-amylase 1 positive surface areas within the glands of mice of the different groups. This aimed to assess the effect of radiation and BMMSCs transplantation on the gland function in accordance to other previous studies ${ }^{(27,33,34)}$.

The forgoing results revealed that the percentage of $\alpha$-amylase 1 positive surface areas within the glands of mice of group II was significantly reduced at all time intervals (D 6, D 30 and D 90) post-radiation, compared with the control group. The glands of mice of group II at day 90 post-radiation showed the lowest value in all groups. These results were consistent with the reported histological results and were in agreement with findings reported by previous investigators ${ }^{(27,34)}$.

The recorded immunohistochemical results and statistical data in this study confirmed the histological results since the percentage of $\alpha$-amylase 1 positive surface areas within the glands of mice of both treated groups (group III and group IV) was significantly increased compared with group II at the different intervals, especially at day 90 post-radiation. However, group IV (irradiated and injected with BM-MSCs at day 11 post-radiation) showed less significant increase compared to group III (irradiated and injected with BM-MSCs $24 \mathrm{~h}$ post-radiation). So, according to this protocol, the time of BM-MSCs transplantation should be considered as a main influencing factor.

\section{CONCLUSIONS}

From the previously mentioned results, the following could be concluded:
1. Intraglandular transplantation of BM-MSCs could successfully regenerate radiation-induced submandibular salivary gland damage and restore the gland function.

2. The early transplantation of BM-MSCs 24 hours post-radiation (i.e. almost with the beginning of early phase of radiation damage) was markedly more beneficial for tissue regeneration and restoration of glands function than the transplantation at day 11 post-radiation (i.e. after the end of the early phase).

3. Intraglandular transplantation of BM-MSCs is considered as a promising treatment for radiation-induced xerostomia.

\section{REFERENCES}

1. Sakai T. Development and regeneration of salivary gland toward for clinical application. Oral Sci Int 2016; 13: 7- 14 .

2. de Almeida P, Grégio A, Machado M, de Lima A and Azevedo L. Saliva composition and functions: a comprehensive review. J Contemp Dent Pract 2008; 9: 72- 80 .

3. Redman R. On approaches to the functional restoration of salivary glands damaged by radiation therapy for head and neck cancer, with a review of related aspects of salivary gland morphology and development. Biotech Histochem 2008; 83: 103- 30 .

4. Thomson W, Lawrence H, Broadbent J and Poulton R. The impact of xerostomia on oral-health-related quality of life among younger adults. Health Qual Life Outcomes 2006; 4: 8- 92 .

5. von Bultzingslowen I, Sollecito T, Fox P, Daniels T, Jonsson R, Lockhart $\mathrm{P}$, et al. Salivary dysfunction associated with systemic diseases: Systematic review and clinical management recommendations. Oral Surg Oral Med Oral Pathol Oral Radiol Endod 2007; 103: S57. e1- 15 .

6. Gill S, Nirola A, Maini A and Sidhu R. Radiation induced salivary gland damage: review on pathogenesis and management. RRJDS 2016; 2: 75- 82.

7. Siegel R, Miller $\mathrm{K}$ and Jemal A. Cancer statistics. CA Cancer J Clin 2016; 66: 7- 30. 
8. Marmary Y,Adar R, Gaska S, Wygoda A, Maly A, Jonathan Cohen J, et. al. Radiation-induced loss of salivary gland function is driven by cellular senescence and prevented by IL6 modulation. Cancer Res 2016; 76: 1170- 80.

9. Wijers O, Levendag P, Braaksma M, Boonzaaijer M, Visch $\mathrm{L}$ and Schmitz P. Patients with head and neck cancer cured by radiation therapy: A survey of the dry mouth syndrome in long-term survivors. Head Neck 2002; 24: 737- 47.

10. Trotti A and Eisbruch A. Reducing xerostomia through advanced technology. Lancet Oncol 2011; 12: 110- 11.

11. Mizrachi A, Cotrim A, Katabi N, Mitchell J, Verheije $\mathrm{M}$ and Friedmanc A. Radiation-induced microvascular injury as a mechanism of salivary gland hypofunction and potential target for radioprotectors. Radiat Res 2016; 186, 189- 95.

12. Vergeer M, Doornaert P, Rietveld D, Leemans C, Slotman $B$ and Langendijk J. Intensity-modulated radiotherapy reduces radiation-induced morbidity and improves health-related quality of life: results of a nonrandomized prospective study using a standardized follow-up program. Int J Radiat Oncol Biol Phys 2009; 74: 1- 8.

13. Beetz I, Steenbakkers R, Chouvalova O, Leemans C, Doornaert P, van der Laan B, et al. The QUANTEC criteria for parotid gland dose and their efficacy to prevent moderate to severe patient-rated xerostomia. Acta Oncol 2014; 53: 597- 604 .

14. Jakobi A, Bandurska-Luque A, Stutzer K, Haase R, Lock S, Wack L, et al. Identification of patient benefit from proton therapy for advanced head and neck cancer patients based on individual and subgroup normal tissue complication probability analysis. Int J Radiat Oncol Biol Phys 2015; 92: 1165- 74 .

15. Grundmann O, Mitchell G and Limesand K. Sensitivity of salivary glands to radiation: from animal models to therapies. J Dent Res 2009; 88: 894- 903.

16. Teshima K, Murakami R, Yoshida R, Nakayama H, Hiraki A, Hirai T, et al. Histopathological Changes in Parotid and Submandibular Glands of Patients Treated with Preoperative Chemoradiation Therapy for Oral Cancer. J Radiat Res 2012; 53, 492- 6.

17. Stramandinoli-Zanicotti R, Sassi L, Schussel J, Torres M, Funchal M, Smaniotto G, et al. Effect of fractionated radiotherapy on the parotid gland: an experimental study in Brazilian minipigs. Int arch otorhinolaryngol 2013; 17: 163- 7 .
18. Muhvik- Urek M, Bralic M, Toma J, Borcic J, Uhac I, Glazar I, et al. Early and late effects of X-irradiation on submandibular gland: a morphological study in mice. Arch Med Res 2005; 36: 339- 43.

19. Bralic M, Muhvic-Urek M and Stemberga V. Cell death and cell proliferation in mouse submandibular gland during early post irradiation phase. Acta Med Okayama. 2005; 59: 153- 9 .

20. Jensen D, Oliveri R, Trojahn S, Nielsen A, Specht L, Bardow A, et al. Mesenchymal stem cell therapy for salivary gland dysfuncion and xerostomia: a systemic review of preclinical studies. Oral Med 2014; 3: 335- 42.

21. Khalil $\mathrm{N}$ and Zulhemma J. Potential stem cell therapy of radiation induced salivary gland dysfunction (A review). Curr Sci Int 2016; 5: 175- 85.

22. Lombaert I, Mohammad M, Movahednia C, Adine C and Ferreira J. Concise review: salivary gland regeneration: therapeutic approaches from stem cells to tissue organoids. STEM CELLS 2017; 35: 97- 105.

23. Li M and Ikehara S. Immunomodulatory properties and therapeutic application of bone marrow derived-mesenchymal stem cells. J Bone Marrow Res 2013; 4: 1- 5.

24. Kalra K and Tomar P. Stem cell: basics, classification and applications. AJPCT 2014; 2: 919- 30.

25. Pham P. Stem cells in clinical applications. Springer International Publishing Switzerland, P.V. Pham (ed.), Stem Cell Processing; 2016.

26. Schwarz S, Huss R, Schulz-Siegmund M, Vogel M, Brandau S, Lang S, et al. Bone marrow-derived mesenchymal stem cells migrate to healthy and damaged salivary glands following stem cell infusion. Int J Oral Sci 2014; 6, 154- 61 .

27. Lim J, Yi T, Choi J, Jang Y,Lee S, Kim H et al.Intraglandular transplantation of bone marrow-derived clonal mesenchymal stem cells for amelioration of post-irradiation salivary gland damage. Oral Oncol 2013; 49: 136- 43.

28. Snykers S, Vanhaecke T, and Rogiers V. Isolation of Rat Bone Marrow Stem Cells, from Methods in Molecular Biology, vol. 320: Cytochrome P450 Protocols: Second Edition 2006; p 265- 72. Edited by: I. R. Phillips and E. A. Shephard (C) Humana Press Inc., Totowa, NJ.

29. Dvorakova J, Hruba A, Velebny V and Kubala L. Isolation and characterization of mesenchymal stem cell population entrapped in bone marrow collection sets. Cell Biol Int 32 2008; 1116- 25. 
30. Fish E, Brown M, Danneman P and Karas A. Anesthesia and Analgesia in Laboratory Animals: Second Edition 2008; American College of Laboratory Animal Medicine Series, Academic Press, San Diego.

31. Flecknell P. Laboratory Animal Anesthesia 3rd Ed 2009; Academic Press, Harcourt Brace Jovanovich, London.

32. Krishnan M, Tennavana A, Saraswathyb S, Sekhric T, Singhd A and Naire V. Acute radiation-induced changes in Sprague-Dawley rat submandibular glands: a histomorphometric analysis. World J Oncol. 2017; 8:45- 52.

33. Zhang N, Huang G, Han Q, Hu X, Yi1 J, YaoL, et al. Functional regeneration of irradiated salivary glands with human amniotic epithelial cells transplantation. Int J Clin Exp Pathol 2013; 6: 2039- 47.

34. Lin C, Chang F, Chen C, Huang C, Hu F, Huang W, et al. Cell therapy for salivary gland regeneration. J Dent Res 2011; 90: 341- 6 .

35. Brandtzaeg P. Mucosal and Glandular Distribution of Immunoglobulin Components; immunohistochemistry with a cold ethanol-fixation technique. Immunol 1974; 26: 1101- 14.

36. Korsrud F and Brandtzaeg P. Quantitative immunohistochemistry of immunoglobulin- and J-chain-producing cells in human parotid and submandibular salivary glands. Immunol 1980; 39: 129- 40. 